\title{
Red Blood Cell Analysis by Hyperspectral Imaging
}

\author{
Hüseyin Kurtuldu ${ }^{*}$ (D) , Aynur Didem Oktan ${ }^{1}$ (D), Hatice Candan ${ }^{1}$ (D), Beste Sahra \\ Cihangiroğlu 1 (iD
}

\begin{abstract}
Hyperspectral imaging is a new technology that aims to use the spectral information of each pixel in different spectral bands to find, identify and classify objects in an image. The hyperspectral imaging system, which is frequently used in the field of remote sensing, is becoming a new imaging model for medical applications and non-invasive disease diagnosis. In this study, a hyperspectral microscope system capable of capturing images of biological samples at different range of spectral wavelengths was developed. With this system, red blood cells in the blood sample were analyzed at various wavelengths and image classification was performed to determine the locations of red blood cells (erythrocytes). Subsequently, the detection of cytoplasm, cell edge, extracellular fluid, and pale area in the cell center of each erythrocyte was successfully performed.

Keywords

Hyperspectral imaging, red blood cell, classification, segmentation, spectral bands

${ }^{1}$ Department of Biomedical Engineering Başkent University

${ }^{*}$ Corresponding Author:hkurtuldu@baskent.edu.tr

Manuscript received date: July 11, 2018

Accept Date: November 27, 2018

Published Date: December 31, 2018.
\end{abstract}

\section{INTRODUCTION}

Blood analysis is one of the most commonly used diagnostic techniques in healthcare. Most microscope-based high-tech systems frequently require blood smearing and evaluation by a pathologist for definitive designation of blood diseases [1]. Anemia, the blood hemoglobin value below the level determined by the World Health Organization [2], causes tissue hypoxia by reducing the oxygen-carrying capacity of blood. The diagnosis of anemia made with the naked eye under a microscope depends on the experience of the individual. For this reason, it is important to develop systems that will eliminate the human factor in such diagnoses.

Three different types of cells are usually imaged in microscopic analyzes leukocytes, erythrocytes, and platelets. The hemoglobin $(\mathrm{Hb})$ concentrations of the blood smear as well as the leukocyte, platelet and erythrocyte counts are also examined when anemia is diagnosed. Erythrocytes are cells that take oxygen from the lungs and transport them to tissues and organs through the $\mathrm{Hb}$ protein for the body and organs. They do not contain mitochondria or nuclei when they mature. The cells are about $7.5 \mu \mathrm{m}$ in diameter and have a thickness at the thickest point of $2.0 \mu \mathrm{m}$ and a minimum thickness of $1 \mu \mathrm{m}$ in the center. Due to their bi-concave structures, the central part of the erythrocyte cell appears paler in the microscopic examination than in the other areas. The ratio of the diameter of this region to the cell diameter is one of the methods used to determine the amount of hemoglobin. Normally, this ratio is $1 / 3$. As it decreases, the amount of tissue hypoxia increases. When the ratio is $1 / 2$, it is termed + hypochromia. If the ratios are $2 / 3$ and $3 / 4$, they are then called ++ hypochromia and +++ hypochromia, respectively. As the diameter of the pale area is very close to the erythrocyte cell diameter, it is named ++++ hypochromia. The correct determination of this ratio is critical for accurate diagnosis [3].

Several methods have previously been applied for blood cell segmentation, including the separation of blood cells into leukocytes and erythrocytes. Sharif et al. [4] provided a method for automatic erythrocyte count. This method included Ycbcr color transformation, masking, and morphological operations with a watershed algorithm. Chourasiya and Rani [5] proposed an efficient and cost-effective computer-based imaging system that performs analysis to automate erythrocyte counting. This technique employed a masking method to identify individual leukocytes and distinguish overlapping ones in the blood sample. Wang et al. [6] visualized blood cells by a scanning electron microscope. Individual cells were extracted using a contour 
guiding method, and the variation of each pixel on a cell image was used to obtain the three-dimensional shape information. Guan et al. [7] used a new method based on hyperspectral imaging for pathological leukocyte segmentation. This technique was used to capture one-dimensional spectral and two-dimensional spatial information of leukocytes. The imaging system consisted of a CCD camera to capture images in a light microscope, an acousto-optic tunable filter for wavelength selection, a computer for analyzing images and spectral curves. By applying a spectral sorting algorithm, leukocytes were divided into four groups at a wavelength range of 550 to $950 \mathrm{~nm}$. Wu et al. [8] offered a gray level-based method that can only detect nuclei to segment leukocytes. Another method proposed by Liao and Deng [9] was limited to multicolored leukocyte cells and, in practice, gave good results in circular leukocyte cells. On the other hand, the two neural network-based methods presented by Yi et al. [10] did not distinguish between the nucleus and the cytoplasm, as they performed well in detecting leukocytes [10]. Until now, there has been no study on the segmentation of erythrocyte cells via hyperspectral imaging.

In this study, to the best of the authors' knowledge, a hyperspectral microscopic imaging system capable of capturing images of blood smear at different spectral wavelengths was used for the first time to determine the location, cytoplasm, cell edge, extracellular fluid and the pale area of erythrocytes. This study suggests that hyperspectral imaging can be used as a useful tool to diagnose blood diseases such as anemia.

\section{MATERIALS \& METHOD}

\subsection{Hyperspectral imaging system}

Hyperspectral imaging measures the intensity of light reflected or transmitted from illuminated materials in a wide range of wavelengths from visible to infrared. The spectral signature of each pixel is obtained for finding, recognizing, and rating objects in an image. Since each material has its own signature, it is possible to separate objects from each other [11,12]. Hyperspectral imaging is becoming a technology used in a wide range of geology, chemistry, medicine, agriculture, forestry, biomedical, space-planet research, and military applications for defense and security purposes [13, 14].

In this study, blood smear samples were examined with an upright Olympus microscope at 100X magnification. A liquid crystal tunable filter (LCTF) attached to a CMOS camera was integrated into the microscope to obtain hyperspectral images. A halogen light source was used for illumination. The block diagram of the system components as well as the microscope system are shown in Figure 1. The LCTF was used to set the wavelength between 420 and $730 \mathrm{~nm}$, and the camera was used to capture gray scale images at the desired wavelength. The camera's exposure (the amount of light reaching the sensor) and gain values (the amplification of the sensor signal) were adjusted to optimize the intensity level of the images for different ranges of wavelength. Increasing the exposure time allows the sensor to collect more light. 


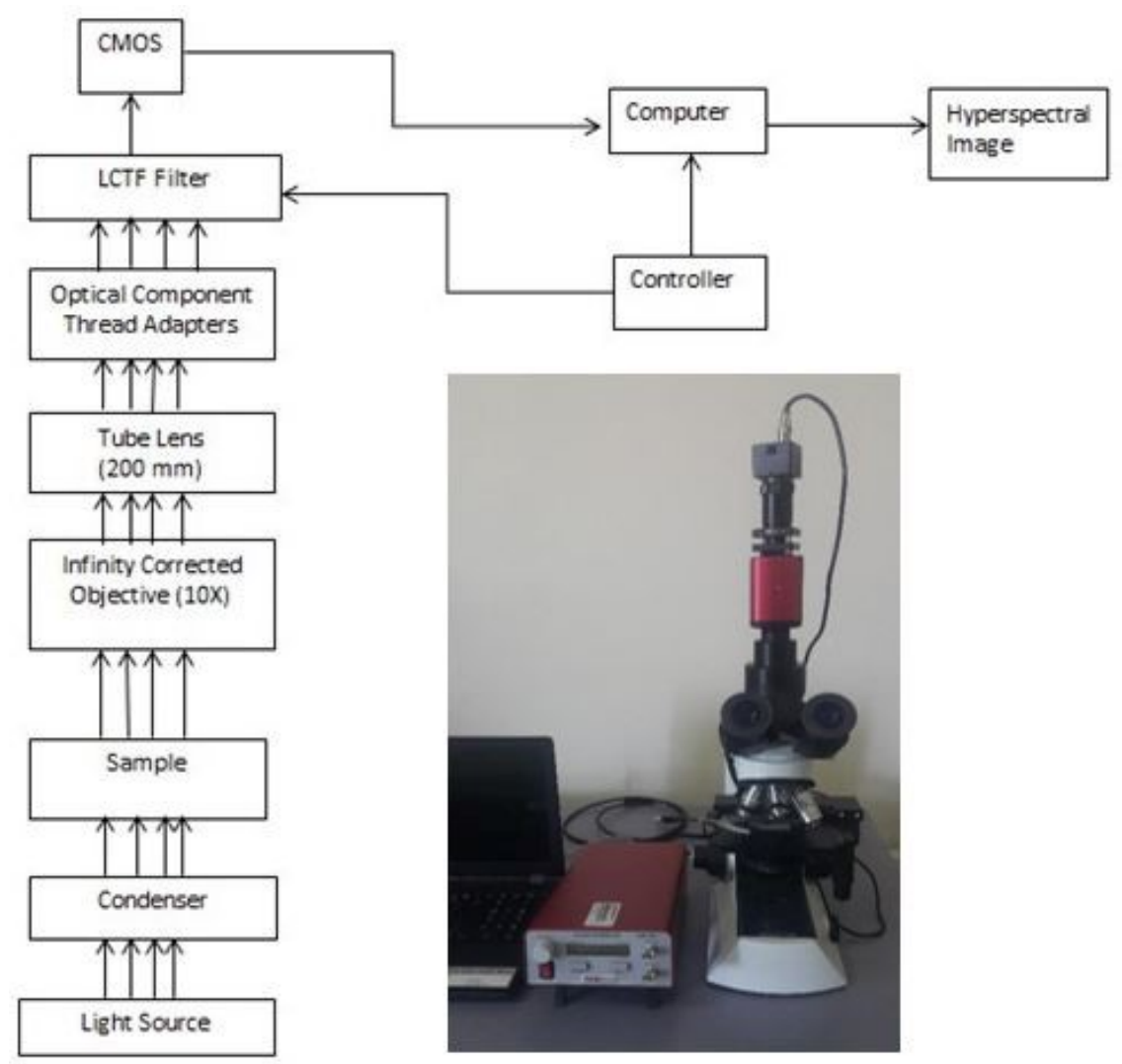

Figure 1. Block diagram of the components for hyperspectral microscope system.

\subsection{Acquisition}

In the range of 500-690 nm with $10 \mathrm{~nm}$ increments, 20 different images of the sample were captured (Figure 2). During the acquisition, gain was kept constant $(1 / 10 \mathrm{~dB})$ and four exposure values were determined between 500 and $690 \mathrm{~nm}$. As listed in Table 1, the exposure value was set to $0.325 \mathrm{~s}$ for the wavelength range of $500-530 \mathrm{~nm}, 0.167 \mathrm{~s}$ for $540-570 \mathrm{~nm}, 0.125 \mathrm{~s}$ for $580-600 \mathrm{~nm}$, and $0.100 \mathrm{~s}$ for $610-690 \mathrm{~nm}$. Since the halogen source produces a more intense light in the infrared portion of the spectrum, the exposure value decreases with increasing wavelength.

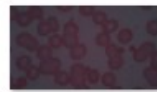

500

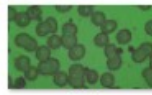

540

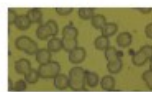

580

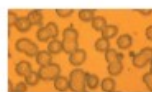

620

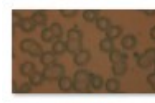

660

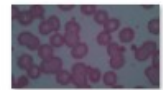

510

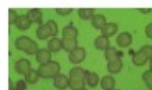

550

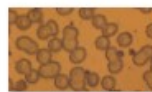

590

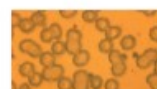

630

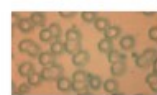

670

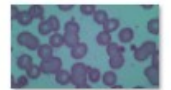

520

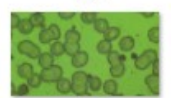

560

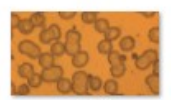

600

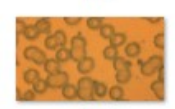

640

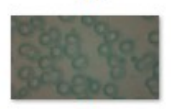

680

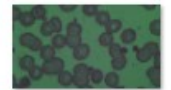

530

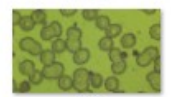

570

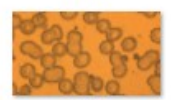

610

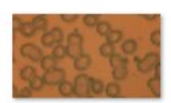

650

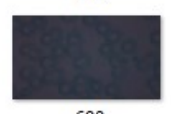

690

Figure 2. Hyperspectral images of the blood sample captured between 500 and $690 \mathrm{~nm}$ with an increment of $10 \mathrm{~nm}$. 
Table 1. Exposure values of the camera for different wavelength ranges.

\begin{tabular}{|l|l|}
\hline Wavelength (nm) & Exposure (s) \\
\hline $500-530$ & 0,325 \\
\hline $540-570$ & 0,167 \\
\hline $580-600$ & 0,125 \\
\hline $610-690$ & 0,100 \\
\hline
\end{tabular}

\subsection{Preprocessing}

Background subtraction was applied to the raw images to compensate for uneven illumination and image artifacts due to optical components in the system. A gray-scale image of the cover-glass blood smear captured at $600 \mathrm{~nm}$ is shown in Figure 3(a). A background image taken on an empty cover-glass at the same wavelength is delineated in Figure 3(b). The images were passed through a Gaussian filter with a 7x7 scan window to reduce noise. The image was normalized by the background after being extracted from the background image. The background-removed blood image is represented in Figure 3(c). A similar procedure was applied to all images captured between 500 and $690 \mathrm{~nm}$.

(a)

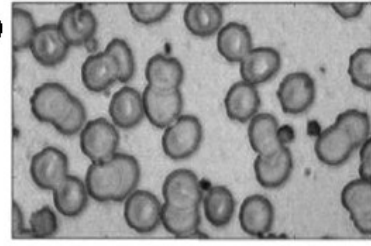

(b)

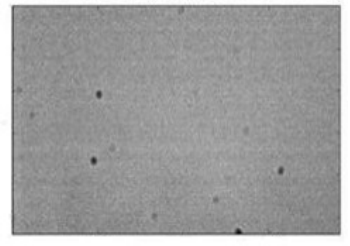

(c)

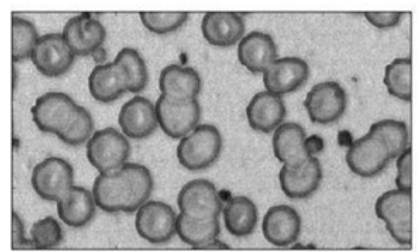

Figure 3. (a) Raw image of the cover-glass blood smear at $600 \mathrm{~nm}$, (b) background image of an empty cover-glass, (c) background subtracted image of the blood smear

\subsection{Classification}

23 different regions of interest in the vicinity of individual erythrocyte cells were selected in blood smear images for classification (Figure 4(a)). Four regions, the central region of erythrocytes (Region-1), cytoplasm (Region-2), cell margins (Region-3), and extracellular fluid (Region-4) were identified for each cell (Figure 4(b)). Figure 5 delineates how the spectral intensity changes between 500 and $660 \mathrm{~nm}$ for these four regions of a single cell. The extracellular region transmits more light than the other regions below $610 \mathrm{~nm}$ while the pale region absorbs less light than the extracellular area above this wavelength. The cell edge has a similar absorbance throughout the wavelength range and the cytoplasm has a transmittance peak at about $570 \mathrm{~nm}$. Images captured above $660 \mathrm{~nm}$ were not used in the classification due to defocusing caused by chromatic aberration and insufficient light. 23 individual erythrocyte cells in blood smear images were selected for classification in these wavelengths. 

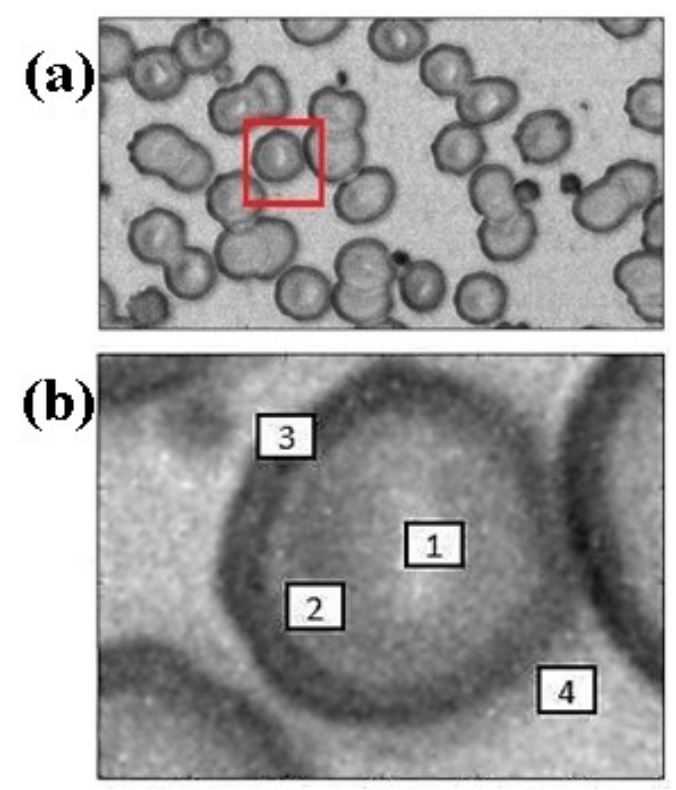

Figure 4. (a) An individual erythrocyte cell selected for analysis (b) identification of the regions-1) pale area 2) cytoplasm, 3) cell edge, and 4) extracellular fluid.

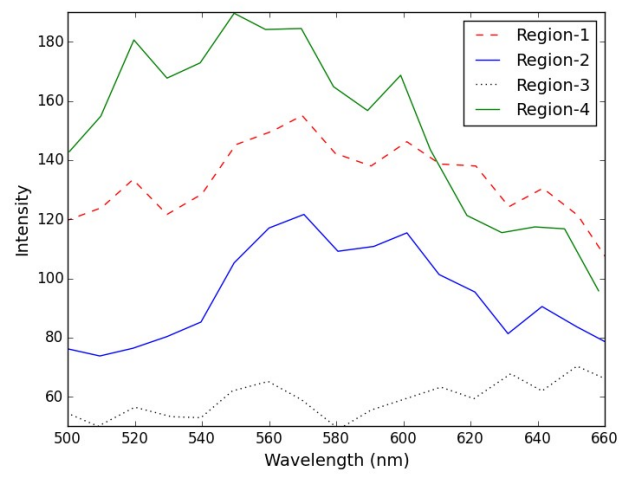

Figure 5. For the regions (Region 1, 2, 3 and 4) shown in Figure 4(b), spectral intensity as a function of wavelength.

Support Vector Machines (SVM), a set of supervised machine learning methods, was employed to determine the optimal level of separation between the regions $[15,16] .10$ of the 23 erythrocyte cells were used as training data and the remaining 13 were utilized as test data. For a total of 17 wavelengths separated by $10 \mathrm{~nm}$, the intensity ranges were determined by considering the pixel values and standard deviations of the four regions within each cell. These ranges were trained in the SVM classifier. The classification was performed pixel by pixel for the region of interest. After the training, the classifier in the selected wavelength predicts which class (region 1, 2,3,4) each pixel in the region belongs to. Increasing the number of training and test samples is expected to increase the performance of classification models.

\section{RESULTS}

Figure 6 shows the result of classifying one of the 13 cells selected for testing. The colors assigned to Region-1 (pale area), Region-2 (cytoplasm), Region-3 (cell edge), and Region-4 (extracellular fluid) are black, dark blue, pink, and white, respectively. Not a single wavelength was sufficient to detect all regions at the same time, so the classification of multiple wavelengths was necessary. While the wavelength between 520 and $540 \mathrm{~nm}$ is more suitable for detecting the pale area, the range of 550 to 630 $\mathrm{nm}$ is better for identifying the extracellular fluid region. 


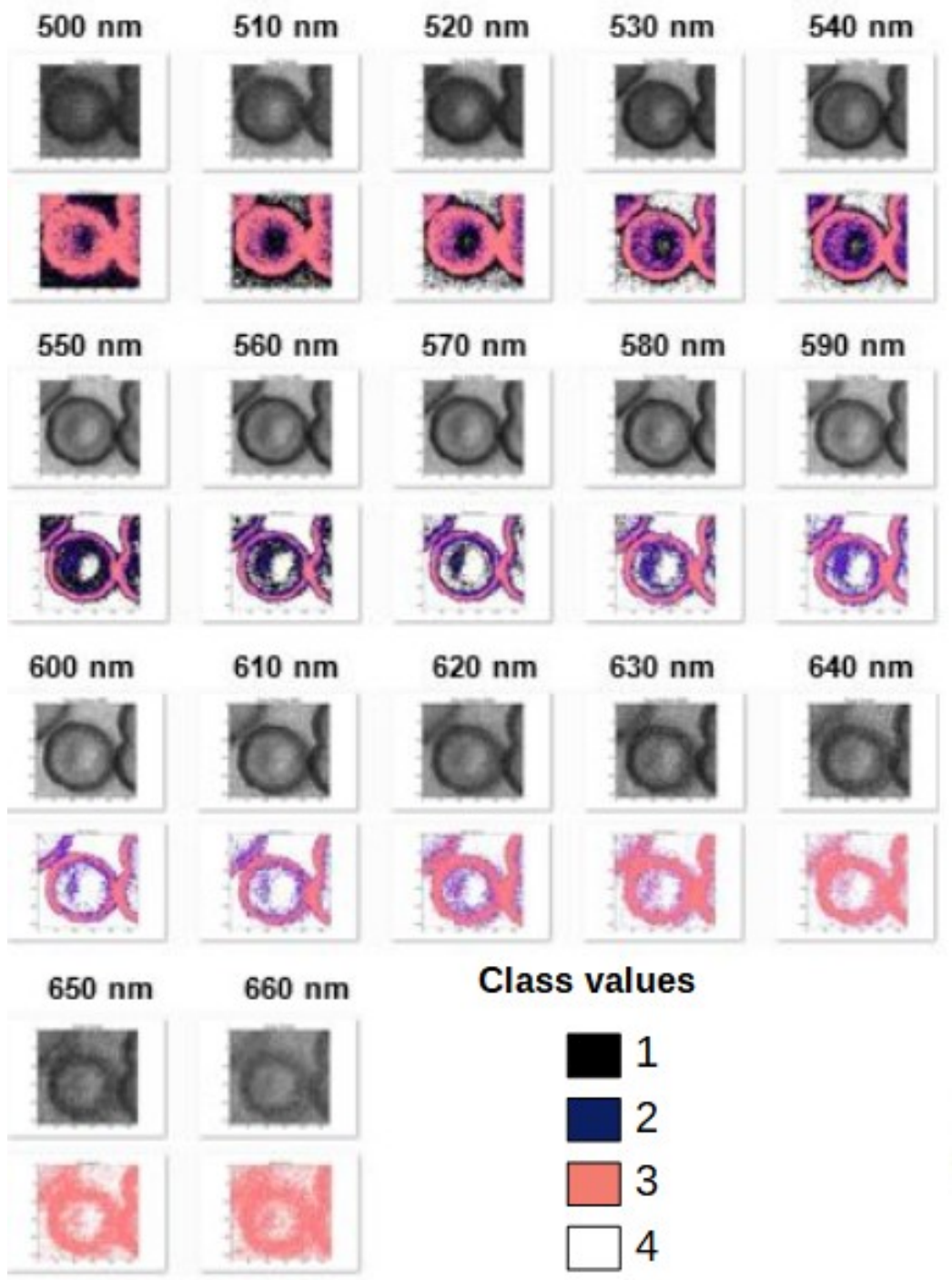

Figure 6. Classification of a cell in a region of interest based on the values Region-1) pale area 2) cytoplasm, 3) cell edge, and 4) extracellular fluid.

The performance of the test with 13 cells was measured by sensitivity and specificity. Test sensitivity is the ability of a test to identify the regions accurately with the assigned region number (true positive rate), whereas the test specificity is the ability of the test to correctly identify those without the appointed number (true negative rate) At the range from 520 to 540 $\mathrm{nm}$, the central region 1 and the region 2 with cytoplasm were detected with $49.7 \%$ and $33.4 \%$ sensitivity, and $56.8 \%$ and $36.3 \%$ specificity, respectively. The region of cell margin had a sensitivity of $90.3 \%$ and specificity of $98.1 \%$ in the range of $550-590 \mathrm{~nm}$. The fourth segment, extracellular fluid fraction, was predicted between wavelengths of 550 and $630 \mathrm{~nm}$ with 96.3 $\%$ sensitivity and $96.6 \%$ specificity. The accuracies of classification were $53.8 \%, 38.4 \%, 92.3 \%$, and $92.3 \%$ for Region- 1,2 , 3 , and 4 , respectively.

\section{DISCUSSION AND CONCLUSION}

In this study, we proposed a hyperspectral microscopic imaging system capable of capturing images of blood smear over wide range of spectral wavelengths. A classifier method trained with intensity images of different wavelengths was used to identify the cytoplasm, the border and the pale area of erythrocyte cells as well as the region of extracellular fluid.

The analysis of erythrocyte cells is important in the diagnosis of anemia, where the level of hypochromia is generally determined by the diameter of the pale area and that of the cells. This method is usually performed by a pathologist, so it is prone to human error. Accurate determination of the level affects the treatment to follow [17]. In this study, we proposed a hyperspectral analysis method that could be used to automate this diagnostic process. In this technique, the border defining the cell pixels was detected with high accuracy. We showed that imaging at wavelengths between 520 and $540 \mathrm{~nm}$ is best for 
distinguishing the number of pixels in the pale area and the pixels belonging to the erythrocyte cell. Training the classifier with more cells will increase the accuracy of the detection of the pale region. Accuracy can also be increased by using a fixed, high transmission band-pass filter in this wavelength range, rather than a tunable filter with a relatively low transmittance.

In clinical applications, the hyperspectral microscopic imaging method can be used to determine the level of hypochrome after the peripheral smear of blood samples from patients. In this way, errors due to the human factor could be eliminated in diameter measurement.

Extracellular fluid contains ions such as sodium, chlorine, and potassium. These ions provide acid-base balance by intracellular osmotic pressure. Hypernatremia and hyponatremia are related to the amount of sodium ion. Sodium has an emission at about $589 \mathrm{~nm}$ [18]. The hyperspectral system, which can detect the extracellular fluid region with accuracy above $90 \%$ at 550-630 nm, could also be used to obtain the spectral intensity as a function of the amount of sodium present in this fraction of the cell.

\section{References}

[1] M. F. Özçelik and H. Ş. Bilge, "The determination of white blood cell borders with using of logical and morphological operations in microscopic blood images," in Signal Processing and Communications Applications Conference (SIU), 2010 IEEE 18th. IEEE, 2010, pp. 688-691.

[2] N. Altin, M. Koray, S. E. Meseli, and H. Tanyeri, "Oral manifestations of anemia," CLINICAL AND EXPERIMENTAL HEALTH SCIENCES, vol. 6, no. 2, pp. 86-92, 2016.

[3] İ. AYDOĞDU, "Hastalıkta ve sağlıkta kan hücreleri," Turkiye Klinikleri Journal of Hematology Special Topics, vol. 5, no. 4, pp. 16-27, 2012.

[4] J. M. Sharif, M. Miswan, M. Ngadi, M. S. H. Salam, and M. M. bin Abdul Jamil, "Red blood cell segmentation using masking and watershed algorithm: A preliminary study," in Biomedical Engineering (ICoBE), 2012 International Conference on. IEEE, 2012, pp. 258-262.

[5] S. Chourasiya and G. U. Rani, "Automatic red blood cell counting using watershed segmentation," Hemoglobin, vol. 14, p. 17, 2014.

[6] R. Wang, B. MacCane, and B. Fang, "Rbc image segmentation based on shape reconstruction and multi-scale surface fitting," in Information Science and Engineering (ISISE), 2010 International Symposium on. IEEE, 2010, pp. 586-589.

[7] Y. Guan, Q. Li, H. Liu, Z. Zhu, and Y. Wang, "Pathological leucocyte segmentation algorithm based on hyperspectral imaging technique," Optical Engineering, vol. 51, no. 5, p. 053202, 2012.

[8] J. Wu, P. Zeng, Y. Zhou, and C. Olivier, "A novel color image segmentation method and its application to white blood cell image analysis," in Signal Processing, 2006 8th International Conference on, vol. 2. IEEE, 2006.

[9] Q. Liao and Y. Deng, "An accurate segmentation method for white blood cell images," in Biomedical Imaging, 2002. Proceedings. 2002 IEEE International Symposium on. IEEE, 2002, pp. 245-248.

[10] F. Yi, Z. Chongxun, P. Chen, and L. Li, "White blood cell image segmentation using on-line trained neural network," in Engineering in Medicine and Biology Society, 2005. IEEE-EMBS 2005. 27th Annual International Conference of the. IEEE, 2006, pp. 6476-6479.

[11] P. Shippert, "Introduction to hyperspectral image analysis," Online Journal of Space Communication, vol. 3, 2003.

${ }^{[12]}$ S. V. Panasyuk, S. Yang, D. V. Faller, D. Ngo, R. A. Lew, J. E. Freeman, and A. E. Rogers, "Medical hyperspectral imaging to facilitate residual tumor identification during surgery," Cancer biology \& therapy, vol. 6, no. 3, pp. 439-446, 2007.

[13] T. H. Kosanke, S. E. Perry, and R. Lopez, "High-resolution hyperspectral imaging technology: Implications for thin-bedded reservoir characterization," 2017.

[14] L. Khaodhiar, T. Dinh, K. T. Schomacker, S. V. Panasyuk, J. E. Freeman, R. Lew, T. Vo, A. A. Panasyuk, C. Lima, J. M. Giurini et al., "The use of medical hyperspectral technology to evaluate microcirculatory changes in diabetic foot ulcers and to predict clinical outcomes," Diabetes care, vol. 30, no. 4, pp. 903-910, 2007.

[15] O. Sezer, A. Erçil, and M. Keskinoz, "Independent component based 3d object recognition using support vector machines," in Signal Processing and Communications Applications Conference, 2005. Proceedings of the IEEE 13th. IEEE, 2005, pp. 99-102.

[16] G. Mercier and M. Lennon, "Support vector machines for hyperspectral image classification with spectral-based kernels," in Geoscience and Remote Sensing Symposium, 2003. IGARSS'03. Proceedings. 2003 IEEE International, vol. 1. IEEE, 2003, pp. 288-290. 
Red Blood Cell Analysis by Hyperspectral Imaging - 16/ 34

[17] B. Turgut, "How important is anemia for the clinician?" Balkan Medical Journal, vol. 2010, no. 1, 2010. 